\title{
Succès concrets du Chronic Care Management en ambulatoire
}

\section{Philippe Giroud ${ }^{a}$, Milo Puhan ${ }^{b}$, Mathias Früh, Daniela Zimmermann-Fehr, Marianne Ehrler}

a Dr rer. pol., b Prof. Dr méd

De nombreux patientes et patients souffrant d'une affection chronique se sentent marginalisés, même en Suisse, alors que celle-ci dispose de l'un des systèmes de santé les meilleurs du monde. Un décalage croissant apparaît entre les besoins d'une population comportant de plus en plus de malades chroniques et ceux d'un système de santé orienté vers la médecine aiguë.

\section{Besoins multiples des personnes atteintes de maladies chroniques}

La pénurie de soins règne là où le besoin se fait le plus sentir. En effet, en Suisse, près d'une personne sur trois souffre d'une maladie chronique à partir de 15 ans. En

\section{Résumé}

En Suisse, près de 2,2 millions de personnes ont des besoins parfois très spécifiques en matière de système de santé, lequel rembourse en premier lieu les maladies, les complications médicales et les séjours hospitaliers. La pénurie règne là où la demande est la plus forte, c'est-à-dire lors de maladies chroniques qui engendrent $80 \%$ des coûts de la santé.

Sur le plan national, la Ligue pulmonaire prend en charge près de 100000 patientes et patients souffrant de maladies chroniques des voies respiratoires et des poumons. Elle donne concrètement l'exemple du Chronic Care Management en ambulatoire à différents niveaux. A l'aide d'une approche de conseil globale, elle obtient ainsi une plus grande qualité de vie chez les personnes concernées, aspire moins d'exacerbations de la maladie, de jours d'hospitalisation et a une performance physique accrue grâce à I'autogestion en cas de BPCO. Elle contribue également à une augmentation significative de la qualité et de l'efficacité lors du traitement de patientes et patients souffrant d'apnée du sommeil. Tout cela est possible uniquement grâce à une collaboration interdisciplinaire avec le corps médical traitant et le personnel spécialisé non médical parfaitement qualifié. Lorsque les nombreuses petites histoires à succès sont ajoutées les unes aux autres, le "tableau fini» du Chronic Care Management apparaît alors plus réel que s'il est perçu séparément. Les initiatives, les projets et les offres qui s'orientent systématiquement vers le bénéfice du patient et vers la qualité du traitement doivent donc être encore plus encouragés à l'avenir. En effet, ce qui compte au final, c'est le résultat à la fin de la chaîne de traitement, soit la qualité de vie et celle des soins. outre, une Suissesse/un Suisse sur cinq âgé(e) de plus de 50 ans est multimorbide, avec une tendance à la hausse. Ce phénomène est alarmant. En effet, vivre avec une maladie chronique a un impact professionnel, familial et donc sociétal. Ce groupe de patients engendre près de $80 \%$ des coûts de la santé [1].

Les besoins multiples nécessitent des informations fiables et compréhensibles. Lorsque celles-ci font défaut, de mauvaises décisions et actions s'ensuivent rapidement, comme par exemple des consultations médicales pouvant être évitées, une aggravation de l'état de santé et, dans les cas sévères, des hospitalisations. Celles-ci conduisent à des absences prolongées sur le lieu de travail ou au sein du ménage. Cela a nécessairement des impacts majeurs sur la qualité de vie des personnes concernées ainsi que sur leur environnement social direct [2].

\section{Le "grand tableau» du Chronic Care Management}

On qualifie également les maladies chroniques d'icebergs vers lesquels le système de santé se dirige à grands pas. La prise en charge de personnes souffrant de maladies chroniques est décrit à juste titre comme l'un des enjeux les plus urgents du XXI ${ }^{\mathrm{e}}$ siècle. Avec un nombre de malades chroniques augmentant de manière fulgurante, l'objectif doit garantir une prise en charge cohérente d'une qualité la plus élevée possible tout en gardant des coûts acceptables [3].

La réussite du Chronic Care Management dépendra avant tout de la qualité de la collaboration entre les différents secteurs et groupes professionnels sur le terrain, et de son interdisciplinarité. En outre, un glisse- 
ment des compétences de décision vers des structures et des professionnels de la santé meilleur marché mais tout aussi compétents est déterminant. La rapidité avec laquelle le dossier électronique du patient se généralisera en Suisse sera décisive, de même que la mise en œuvre d'un renforcement ou d'une indemnisation de l'autogestion. Sera déterminante également, leur pertinence d'un point de vue politique.

\section{Une qualité de vie améliorée grâce à un conseil global}

Une maladie chronique a une influence directe sur la qualité de vie des personnes concernées. C'est pourquoi, parallèlement à l'installation et à la mise en service de l'appareil de thérapie respiratoire, les conseillères et conseillers de la Ligue pulmonaire s'efforcent de mettre tout particulièrement l'accent sur l'intégration de la thérapie dans les activités du quotidien et, si nécessaire, d'aborder également des questions d'ordre psychosocial. En font par exemple partie, la réorganisation du quotidien après le diagnostic, le développement d'une nouvelle image de soi, la confrontation avec la maladie (et la mort), mais également la pratique de stratégies de réduction du stress et de gestion des angoisses et des doutes.

\section{Une maladie chronique a une influence directe sur la qualité de vie des personnes concernées.}

Avec un échantillonnage $(\mathrm{N}=2485)$ de près de 100000 patients pris en charge, l'enquête de satisfaction réalisée au printemps 2017 par la Ligue pulmonaire, démontre par conséquent des résultats réjouissants:

- $78 \%$ des personnes interrogées trouvent que leur état de santé s'est amélioré depuis le début de la thérapie, ou qu'il s'est significativement amélioré.

- $82 \%$ de celles-ci indiquent qu'elles peuvent très bien ou bien intégrer les mesures de traitement des conseillers de la Ligue pulmonaire dans le déroulement de leur journée.

- $88 \%$ des patientes et patients ont reçu un bon ou très bon soutien grâce aux conseils de la Ligue pulmonaire, ce qui leur a permis de gérer le quotidien avec la maladie.

- 71\% des personnes interrogées estiment leur qualité de vie comme étant très bonne ou bonne ces quatre dernières semaines.

En définitive, il n'est pas rare qu'une affection grave et chronique (comme par ex. la BPCO) entraîne des questions d'ordre socio-économique. Dans ce domaine, différentes ligues pulmonaires cantonales s'appuient sur la compétence de leurs propre assistantes et assistants sociaux. Ceux-ci clarifient avec les patientes et les patients et leurs proches leurs droits éventuels d'assurés, fournissent des conseils en matière de budget, soutiennent les personnes concernées en ce qui concerne leur situation professionnelle et/ou de logement ou établissent le lien avec les autorités ou les assurances telles que l'AVS ou l'AI.

\section{Deux tableaux cliniques comportant plus d'un demi-million de personnes touchées}

Selon la complexité et le degré d'évolution de l'affection des voies respiratoires ou des poumons, les patientes et les patients profitent d'un conseil globalisé différencié. En Suisse, on estime que plus de 400000 personnes vivent avec une BPCO et que beaucoup plus de personnes souffrent d'une apnée du sommeil que les 150000 personnes recensées jusqu'à maintenant [4].

\section{De meilleurs résultats grâce à l'autogestion lors de BPCO}

Des programmes encourageant la compétence d'autogestion sont en progression également en Suisse [5]. Ils constituent par ailleurs une revendication essentielle des stratégies nationales, comme par exemple celle de Santé2020 et de la stratégie MNT. «Mieux vivre avec une BPCO» est un programme parfaitement évalué au niveau scientifique dans le cas de cette maladie pulmonaire obstructive chronique, et donc très pertinent pour la Suisse également [6].

Des chercheurs de la Collaboration Cochrane ont publié en 2002, 2007 et 2014 des revues systématiques de littérature qui ont analysé les impacts du coaching en autogestion en cas de BPCO [7]. Ils ont donc trouvé des résultats nettement meilleurs d'un point de vue statistique chez les patientes et les patients qui avaient réalisé ce programme de façon structurée, que chez les patientes et les patients qui avaient été répartis au hasard dans la branche des «Usual-Care» (soins habituels). Concrètement, cela signifie:

- une meilleure qualité de vie,

- un taux d'hospitalisation plus faible,

- une meilleure performance physique,

- un taux plus élevé de vaccination contre la grippe et un taux plus élevé d'arrêt du tabagisme.

Le programme est en œuvre dans de nombreux pays sous les formes les plus diverses et sera déployé en 2018 pour la première fois en Suisse sous une forme ambulatoire à plus large échelle. A ce propos, un groupe interdisciplinaire d'experts constitué de représentantes et de représentants de la Société Suisse de Pneumologie et de la Communauté d'intérêt en physiothérapie 


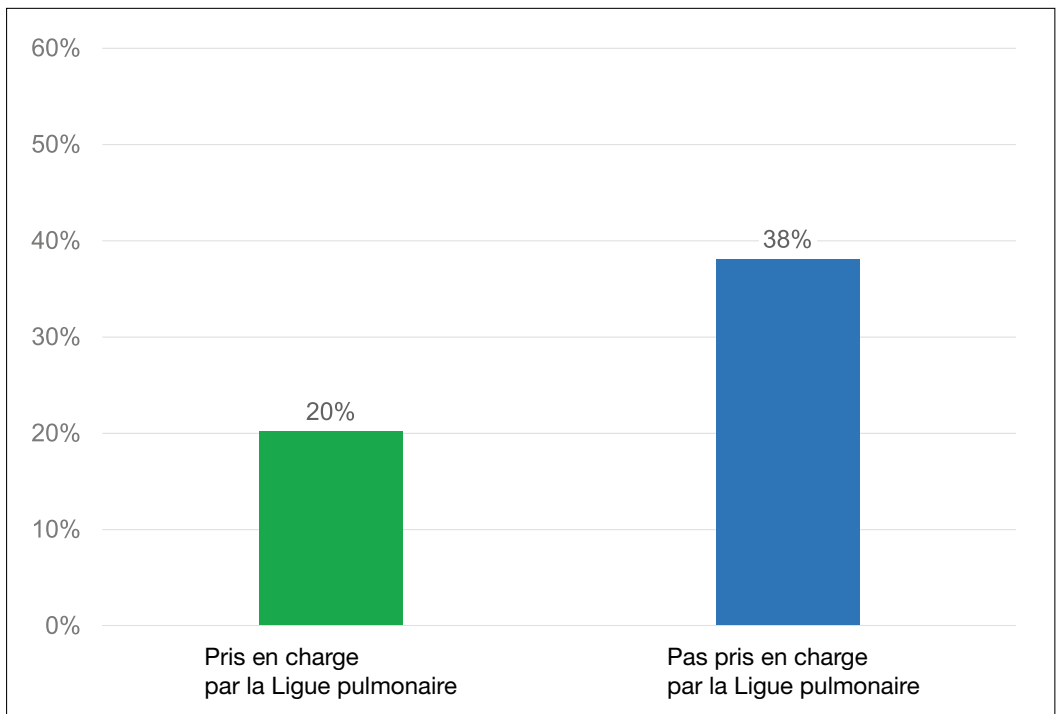

Figure 1: Probabilité d'un arrêt du traitement CPAP. médicales dont bénéficient les patientes et les patients dans l'ensemble du secteur de la santé. Par ailleurs, l'assurance maladie a analysé la qualité du traitement sous un autre angle, en se demandant quelle était la probabilité d'arrêter la thérapie et d'être hospitalisé. Il ressort de l'analyse que le travail de la Ligue pulmonaire a un impact avéré. Les patientes et patients CPAP pris en charge par la Ligue pulmonaire ont donc engendré globalement $10 \%$ de coûts de la santé en moins que les personnes touchées qui ne sont pas prises en charge par la Ligue pulmonaire. Les frais médicaux des personnes prises en charge par la Ligue pulmonaire sont en moyenne inférieurs de 900 francs suisses par an. Helsana attribue cette réduction de coûts à moins de consultations médicales chez les patientes et patients de la Ligue pulmonaire, car les consultations auprès du médecin de premier recours sont en moyenne inférieures de $2 \%$ et celles auprès du médecin spécialiste le sont même de $26 \%$.

En outre, l'analyse indique que les patientes et les patients CPAP tirent profit de la prise en charge par la Ligue pulmonaire également en matière de qualité de leur traitement. La probabilité d'une hospitalisation est inférieure de $12 \%$ chez les personnes prises en charge par la Ligue pulmonaire. Comme le montre la figure 1, les personnes prises en charge par la Ligue pulmonaire interrompent leur thérapie deux fois moins souvent au cours des douze premiers mois. Comme l'ont montré les 1501 cas étudiés en détail, la probabilité d'un arrêt sans prise en charge par la Ligue pulmonaire se situe à $38 \%$ alors qu'elle n'est que de seulement $20 \%$ chez les patients pris en charge par la Ligue pulmonaire.

Le travail à but non lucratif de la Ligue pulmonaire a un impact positif pour les patientes et les patients qu'elle prend en charge, aussi bien en termes d'efficacité que de qualité. Il semble donc que la Ligue pulmonaire endosse le rôle de "Gatekeeper" pour ses patientes et patients, comme les médecins de famille dans les modèles de Managed Care, et contribue donc à un gain en efficacité et en qualité dans le système de la santé.

\section{Des conseillers bien formés pour une meilleure prise en charge}

Le personnel spécialisé non médical est responsable des différences en matière d'efficacité et de qualité. Ce sont eux qui trient, conseillent et coordonnent les différents acteurs, et qui gèrent les interfaces entre ces derniers. Ce faisant, ils sont toujours à la recherche de la solution optimale pour la patiente et le patient individuels.

La Ligue pulmonaire a mis en place une large offre de 
formation continue approfondie, qui a pour objectif aussi bien une spécialisation dans les maladies des voies respiratoires et des poumons que dans les compétences pour des conseils se basant sur l'entretien motivationnel. En collaboration avec la Société Suisse de Pneumologie et la Société Suisse de Pneumologie pédiatrique, la Ligue pulmonaire est en outre responsable de l'examen professionnel «conseiller/conseillère en affections respiratoires et tuberculose» avec certificat fédéral, reconnu par le Secrétariat d'Etat à la formation, à la recherche et à l'innovation.

Des structures compétentes sont mises en place, par le biais de ces formations continues spécialisées et diplômes professionnels, et des gains en termes d'efficacité sont réalisés pour le système de la santé grâce à la sollicitation renforcée de ces structures (comme on le voit dans l'exemple de l'apnée du sommeil), et ceci, sans que des compromis doivent être faits au détriment de la qualité.

\section{De petites avancées pour un grand projet}

Afin de prendre en charge de façon rentable les patientes et les patients multimorbides et souffrant de maladies chroniques et de leur offrir la meilleure des qualités de vie possible dans leur environnement familial, des structures ambulatoires professionnelles sont nécessaires ainsi que des soins globaux quotidiens et des services sociaux avec un accent placé sur l'autogestion, sur un remboursement suffisant des services de conseil et de prise en charge, et sur une collaboration bénéfique et interdisciplinaire [10].

Le mot d'ordre à venir est donc le suivant: «from cure to care» (de la guérison aux soins) [1]. A la place de l'orientation primaire sur les soins aigus qui a pour objectif le rétablissement rapide des patients, une prise en charge globale en réseau et de qualité élevée passe ainsi au premier plan. Elle se focalise sur l'individu en tant que tel et sur ses besoins personnels, avec l'objectif d'optimiser la qualité de vie de la personne malade. Ce mot d'ordre devrait servir de fer de lance à tous les acteurs prenant en charge les malades chroniques.
Liens relatifs aux groupes d'intérêts

Dr rer. pol. Philippe Giroud, responsable du secteur Conseil intégré, Ligue pulmonaire suisse

Prof. Dr méd. Milo Puhan, directeur de l'Institut d'épidémiologie, biostatistique et prévention, Université de Zurich; Membre du Comité de l'association Lunge Zürich et membre du Comité central de la Ligue pulmonaire suisse

Mathias Früh, responsable Pharmaceutique \& Technique médicale, Helsana

Daniela Zimmermann-Fehr, responsable Achat de prestations,

Helsana

Marianne Ehrler, responsable des négociations, gestion des processus et conventions, Communauté d'achat HSK

\section{Remerciements}

Les auteures et auteurs remercient toutes les pionnières et tous les pionniers en Chronic Care en Suisse ainsi que tous les acteurs innovateurs et engagés sans relâche dans l'ensemble des domaines du système de la santé. Qu'ils continuent de contribuer à l'amélioration croissante des soins des personnes souffrant de maladies chroniques.

\section{Crédit graphique}

Helsana Assurances SA

\section{Références}

1 Observatoire suisse de la santé (éd.). Santé en Suisse - Accent sur les maladies chroniques. Rapport national sur la santé 2015. Berne: Hogrefe Verlag; 2015

2 Benz D, Homann B. Die chronisch Vernachlässigten. Beobachter, à l'URL suivante: https://www.beobachter.ch/politik/chronisch-kranke-die-chronisch-vernachlassigten (28 octobre 2017).

3 Steurer-Stey C. Chronische Krankheiten - die neue «Epidemie» des 21. Jahrhunderts. Care Management. 2009;2(4):5.

4 Heinzer R, Vat S, Marques-Vidal P, Marti-Soler H, Andries D, Tobback N, et al. Prevalence of sleep-disordered breathing in the general population: the HypnoLaus study. Lancet Respir Med. 2015;3(4):310-8.

5 Ebert S, Peytremann-Bridevaux I, Senn N. Les programmes de prise en charge des maladies chroniques et de la multimorbidité en Suisse. Obsan Dossier 44. Neuchâtel: Observatoire suisse de la santé.

6 Dalla Lana K, Pfister A, Stoller S, Huber F, Senn O, Steurer-Stey C. Besser Leben mit COPD. Care Management. 2010;3(6):24-7.

7 Zwerink M, Brusse-Keizer M, van der Valk PD, Zielhuis GA, Monninkhof EM, van der Palen J, et al. Self-management for patients with chronic obstructive pulmonary disease. Cochrane database Syst Rev. 2014;19:3.

8 Steurer-Stey C, Dalla Lana K, Braun J, ter Riet G, Puhan M. Effects of the "Living well with COPD" intervention in primary care: a com parative study. ERJ. 2018;51:1701375; DOI: 10.1183/13993003.013752017.

Carron T, Brideveaux P-O, Lörvall K, Parmentier R, Moix J-B, Bey trison $\mathrm{V}$, et al. Feasibility, acceptability and effectiveness of integrated care for COPD patients: a mixed methods evaluation of a pilot community-based programme. Swiss Med Wkly. 2017;147:w14567; DOI: 10.4414/smw.2017.14567.

9 Früh, M, Zimmermann-Fehr D. CPAP-Atemtherapie-Bessere Ergebnisse dank intensiver Betreuung. Helsana-Standpunkt. 2017;2:10-1.

10 Haslbeck J, Klein M, Bischofberger I, Sottas B. Leben mit chronischer Krankheit. Die Perspektive von Patientinnen, Patienten und Angehörigen (Obsan Dossier 46). Neuchâtel: Observatoire suisse de la santé. 